\title{
Why the Dr. Budwig Flaxseed Diet should be Part of your Cancer Treatment Program
}

\author{
Lloyd Arthur Jenkins* \\ Teaching \& Public Speaking, Spain \\ *Corresponding author: Lloyd Arthur Jenkins, Teaching \& Public Speaking, Spain

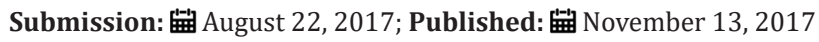

Opinion

Dr. Budwig mixed the flaxseed oil with Quark (a type of German cottage cheese) together and then added fresh ground flaxseeds to the mixture. She had her cancer patients eating this mixture twice a day and reported outstanding results with all types of cancer. She definitely was way ahead of her time in using flaxseeds for cancer treatments.

\section{Clinical Study using Dr. Budwig's Flaxseed Diet}

In a 2005 study report in the International Journal of Cancer an experiment was done were they feed rats any combination of flax extracts tumor growth rate was significantly lower in all of the flaxseeds fed rats Due to decreased cell proliferation and increased apoptosis; Lung metastasis incidence was reduced up to $70 \%$ by all treatments; distant lymph node metastasis was significantly decreased (52\%). Total metastasis incidence was lowered (42\%) in the lignan combined with flax oil fed rats [1].

In the November 2004 issue of Clinical Cancer Research, a study was done combining flaxseed with tamoxifen and alone. The study reported: They used mice with human breast cancer tumors with and without supplemental estrogen. At low estrogen levels, predictive of a woman in menopause, tumors in the flaxseed fed mice shrunk $74 \%$. The tamoxifen produced a similar effect to the flaxseed shrinking the tumors initially, but by the end of the experiment, the tumors had returned to their initial size using just tamoxifen.

Combining tamoxifen and flaxseed shrunk the tumor half again as much as tamoxifen alone did. In the mice kept at high estrogen levels, modeling a premenopausal breast cancer patient, the flaxseed alone inhibited tumor growth by $22 \%$. The tamoxifen alone inhibited tumor growth by $41 \%$ and both together by $50 \%$. Tamoxifen increases the risk of uterine cancer in humans by eight times [2]. It caused the uterus of these test mice to swell by $39 \%$. Flax seed prevented the tamoxifen from causing this uterine growth. Flaxseed on its own inhibited estrogen receptor positive breast cancer and increased the effectiveness of tamoxifen and possibly prevents uterine cancer, a known risk associated with using this drug treatment.

In 2002 Chen et al writing in Nutrition and Cancer a study was done were by scientists injected human breast cancer cells into

mice. Half the mice were given flax seed. Lung metastasis was $56 \%$ in the control group and only $22.2 \%$ in the flax fed mice. Flaxseed decreased lymph node metastasis from $89 \%$ to $33 \%$. Lung tumors decreased by $82 \%$. The researchers showed that these effects were partly due to flax seeds effect of decreasing insulin-like growth factor I and epidermal growth factor receptor expression [3].

Dabrosin et al published in Cancer Letters a study in November 2002 suggesting that flaxseed inhibited angiogenesis in breast tumors by decreasing levels of (VEGF) which is a key factor in breast cancer angiogenesis.

\section{The Study Reported}

The women who eat the most flax lowered their risk of getting breast cancer by $62 \%$ compared to women who do not eat it. Women with this genetic marker who eat large amounts of flax lignans reduce their risk of breast cancer by up to $70 \%[4,5]$.

\section{The Dr. Budwig Natural Cancer Diet}

Those on a cancer healing journey who use the famous flaxseed oil and cottage cheese mix that Dr. Budwig developed can greatly enhance their results. Consuming the flaxseed oil and cottage cheese mixture produces natural oxygen that provides not only a noticeable increase in your energy and strength to your immune system, but it also helps weaken cancer cells. Of course, you can simply grind up flaxseeds in a coffee grinder and add them to a smoothie or to a fresh garden salad, however better still would be to soak the flaxseeds overnight and blend them along with some raw honey using an upright stick blender and spread the mixture on toast or add to a smoothie.

However, be careful not to use any artificial oxygen therapies and vitamin $\mathrm{C}$ or other vitamin cocktails by IV's at the same time as the two therapies will cancel each other out. Therefore, if you do use these therapies, it is best to consume the flaxseed oil and cottage cheese (Quark) after your therapies are complete. In addition to consuming the flaxseed oil and cottage cheese or Quark our patients also consume generous amounts of fruit, vegetables, and plant based foods. White refined sugar, fructose, corn syrup and artificial sweeteners such as Aspartame, Sweet n low, etc., are like fertilizer to cancer and so are removed from the Budwig diet. 
Refined oils and deep-fried foods, store bought pastries are also all removed from their diet. Dr. Budwig found that refined vegetable oils, margarine and deep-fried foods literally suffocated the cells and disabled their healthy electrical charge present in cells. It was for this discovery and the combining of flaxseed and Quark that she was nominated 7 times for the Nobel Prize.

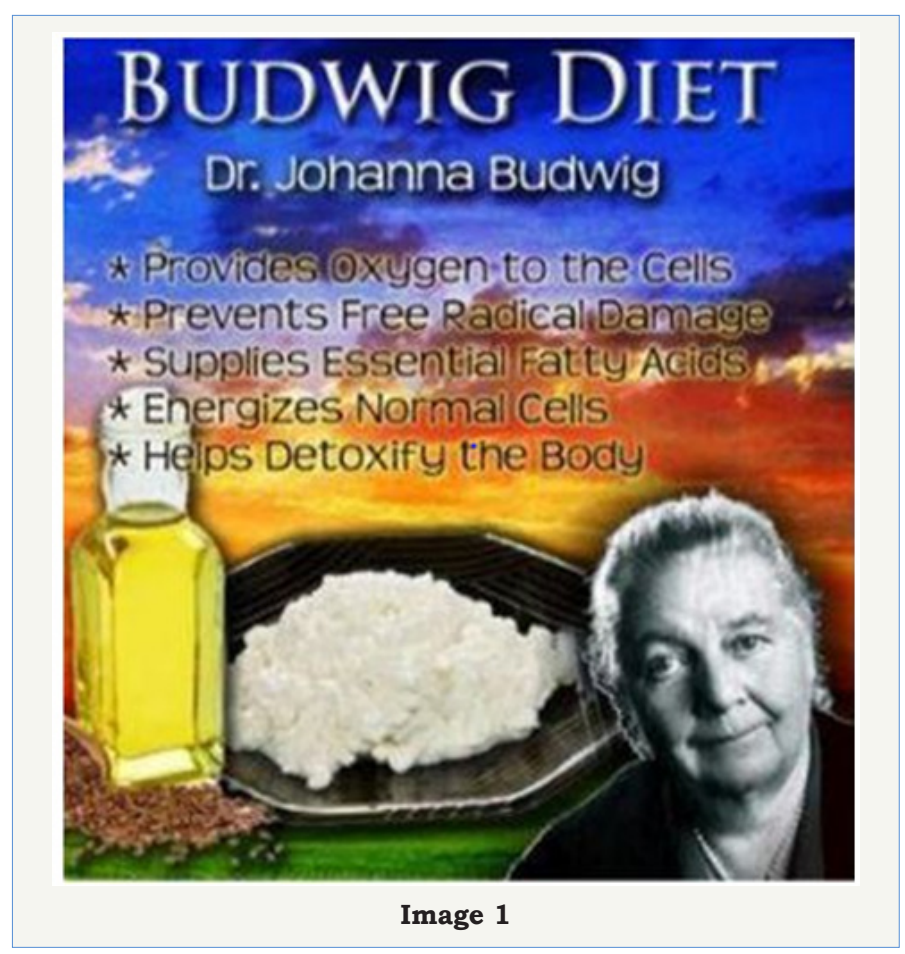

\section{Cancer is on the rise!}

The World Cancer Report predicts new cancer cases will double within two decades. You will obtain better results by simply adding flaxseed to the cancer protocol, as was clearly proven in several clinical studies.

IF you are interested in our free guide that answers over 20 common questions about the famous Dr. Johanna Budwig diet, download it at: www.BudwigCenter.com

And for more information on our clinical and home distance programs, email us: Admin@BudwigCenter.eu (Image 1).

\section{References}

1. Wang (2005) The inhibitory effect of flaxseed on the growth and metastasis of estrogen receptor negative human breast cancer xenograftsis attributed to both its lignan and oil components. Int J Cancer 116(5): 793-798.

2. Chen (2004) Dietary Flaxseed Enhances the Inhibitory Effect of Tamoxifen on the Growth of Estrogen-Dependent Human Breast Cancer (MCF-7) in Nude Mice. Clin Cancer Res 10(22): 7703-7711.

3. (2002) Nutr Cancer 43(2): 187-192.

4. McCann SE, Muti P, Vito D, Edge SB, Trevisan M, Freudenheim JL (2004) Dietary lignan intakes and risk of pre- and postmenopausal breast cancer. Int J Cancer 111(3): 440-443.

5. http://www.denvernaturopathic.com/news/flaxseeds.html 